\title{
The Effect of Curing Conditions on the Electrical Properties of an Epoxy Resin
}

\author{
Istebreq A. Saeedi, Alun S. Vaughan, Thomas Andritsch and Suvi Virtanen \\ The Tony Davies High Voltage Laboratory \\ University of Southampton \\ Southampton, UK, SO17 1BJ \\ iahs1e13@soton.ac.uk
}

\begin{abstract}
Epoxy resins have been used in many high voltage applications like cable terminations and cast resin transformers, where the performance of the associated devices depends critically on the effectiveness of the epoxy insulation. The curing process is key to epoxy electrical performance and, therefore, it is necessary to understand the effect of variations of curing conditions on the electrical properties of the epoxy insulation that results. The work described here is focused on studying the effect of the curing conditions of an epoxy resin, based upon measurements of the influence of curing protocol on the AC breakdown strength, DC conductivity, $T_{g}$ and permittivity of a bisphenol-A system. This research is of potential significance for understanding the behavior of the epoxy resin, because it evaluates the extent to which various curing conditions - and hence network structures - can affect properties.
\end{abstract}

\section{INTRODUCTION}

The thermal, electrical and mechanical properties of thermosetting composites will depend on the resin monomer's molecular structure, the type of hardener used, on the chosen stoichiometry and the degree of curing. In the case of thermosetting epoxy resin systems, it has been shown [1] that the degree of curing depends on the time and temperature of curing. As the curing time increases, so the glass transition temperature, $T_{g}$, shifts to higher values. This increase in $T_{g}$ has been attributed to variation in the molecular dynamics during the curing process, which results in system with a high crosslink density [2]. The resulting crosslink density impacts the physical properties of epoxy resin, as systems with increased crosslinking have improved mechanical properties [3]. Such variations in the properties of epoxy resin systems are, ultimately, caused by variation in both the time and temperature of curing [1-3].

While the influence of curing conditions on the thermal and mechanical properties of epoxy resin is well studied, less attention has been focused on the effect of the cure time and cure temperature on electrical characteristics. It was therefore the objective of the work described here to investigate the effect of curing time on the electrical properties of a DGEBA epoxy resin, where factors such as the curing temperature and the hardener/resin stoichiometry were kept constant. Room temperature measurements of the AC breakdown strength, DC conductivity and complex permittivity have been conducted. The importance of the glass transmission temperature on these properties has been established.

\section{EXPERIMENTAL}

\section{A. Materials}

Diglycidyl ether of bisphenol-A epoxy resin DER332 supplied by Sigma Aldrich, was used for this study, together with the amine hardener Jeffamine D-230 (Huntsman). The resin has an epoxide equivalent molar mass of $172-176 \mathrm{~g} \mathrm{~mol}^{-1}$ and the amine hardener has a hydrogen equivalent molar mass of $60 \mathrm{~g}$ $\mathrm{mol}^{-1}$. The theoretical resin : hardener stoichiometry is therefore 100 : 34.4 parts by mass; this value was kept unchanged throughout this study to maintain complete reaction between the resin epoxide groups and the amine groups of the hardener.

\section{B. Sample Preparation}

For this study, samples nominally $200 \mu \mathrm{m}$ in thickness, were produced by weighting out the calculated theoretical stoichiometric ratio $100: 34.4$ of resin : amine hardener. The resin was preheated at $50{ }^{\circ} \mathrm{C}$ for at least $1 \mathrm{~h}$ to reduce its viscosity. The resin and hardener were then mixed for $5 \mathrm{~min}$ using a magnetic stirrer, after which, the mixture was place in a vacuum oven and degassed until no bubbles could be seen in the mixture. After degassing, the resin/hardener mixture was cast in steel molds, which were place in a fan oven to cure. One set of samples were cured at $80{ }^{\circ} \mathrm{C}$ for between $1 \mathrm{~h}$ and 9 $\mathrm{h}$, then left to cool down to room temperature overnight. A second set of samples was all cured for $2 \mathrm{~h}$ at $80{ }^{\circ} \mathrm{C}$, then cooled down overnight before, finally, being post-cured at 125 ${ }^{\circ} \mathrm{C}$ for different curing time $(1-3 \mathrm{~h})$ and left to cool. After production, all samples were stored in vacuum until used.

Samples used for dielectric spectroscopy were sputtered coated with gold to give a circular gold electrode, $30 \mathrm{~mm}$ in diameter, which ensured good contact between the spectrometer and the sample.

\section{Material Characterization}

Samples were characterized using four analytical techniques. Room temperature measurements of the dielectric properties were conducted using a Solartron 1296 dielectric interface linked to a Schlumberger SI 1260 impedance gain-phase analyzer. The dielectric spectra were measured as a function of frequency from $0.1 \mathrm{~Hz}$ to $0.1 \mathrm{MHz}$, using an applied $\mathrm{AC}$ voltage of amplitude $1 \mathrm{~V}$. AC ramp breakdown testing was conducted using a Phenix Type 600C set operating at $50 \mathrm{~Hz}$; 
the applied AC voltage was increased at the rate of $50 \mathrm{~V} / \mathrm{s}$ until failure. All samples were tested between $6.3 \mathrm{~mm}$ ball bearing electrodes, immersed in silicon oil to prevent flashover. A Perkin Elmer DSC7 differential scanning calorimetry (DSC) was used to evaluate the glass transition of the various systems studied. Heating scans were conducted from $20{ }^{\circ} \mathrm{C}$ to $170{ }^{\circ} \mathrm{C}$ using a heating rate of $10{ }^{\circ} \mathrm{C} \mathrm{min}{ }^{-1}$. Finally, the DC conductivity was measured using a Keithley 6487 picoammeter which was connected to sample holder incorporating $20 \mathrm{~mm}$ circular electrodes. Data were collected for $3 \mathrm{~h}$ at a constant applied DC field of $40 \mathrm{kV} / \mathrm{mm}$.

\section{RESULTS}

\section{A. Thermal Analysis}

The DSC measurements on epoxy resin samples directly after curing at $80{ }^{\circ} \mathrm{C}$, for different curing times, are shown in Fig. 1. As the curing time increase, $T_{g}$ shift to higher values. However, after $6 \mathrm{~h}$ of curing, this trend stops. The increased curing time could have resulted in highly cross linked system. As the cross link density changing, the behavior of $T_{g}$ is affected. This behavior is not anticipated for homogenous systems, and is usually expected for inhomogeneous systems [4].

\section{B. Dielectric Spectroscopy}

The dielectric response of the cured and post cured samples is shown in Fig. 2 and Fig. 3, respectively. Fig. 2 indicates a strong variation in both real and imaginary parts of permittivity of samples cured for different curing time. This behavior perhaps could be explained by the existence of two factors which are namely the degree of crosslinks (i.e. the extent of reaction) and environmental constraints on the movement of the hydroxyether groups resulted from the formation of high density system.

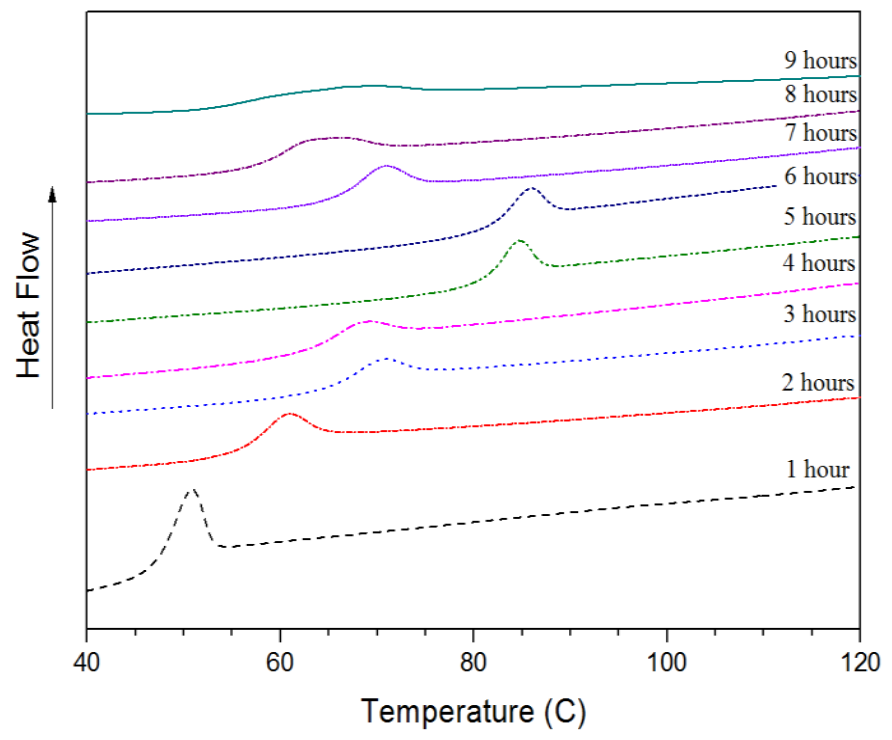

Fig. 1. Differential scanning calorimetry measurments for epoxy resin systems cured at $80^{\circ} \mathrm{C}$ for different curing hours.
As reaction proceeds more hydroxyether groups are formed but, as the crosslink density increases, they become increasingly constrained. Initially, the former dominates, where the increased formation of hydroxyether groups resulted in rising peak of the dielectric $\beta$-relaxation, indicated at the high frequency above $10^{4} \mathrm{~Hz}$ of imaginary permittivity in Fig. 2 (b). However, later on, it's the environmental constraints which dominates; Therefore, the movement of hydroxyether groups become constrained which was reflected by the decreased $\beta$-relaxation after 6 hours of curing, as shown in Fig. 2 (b) and (c).
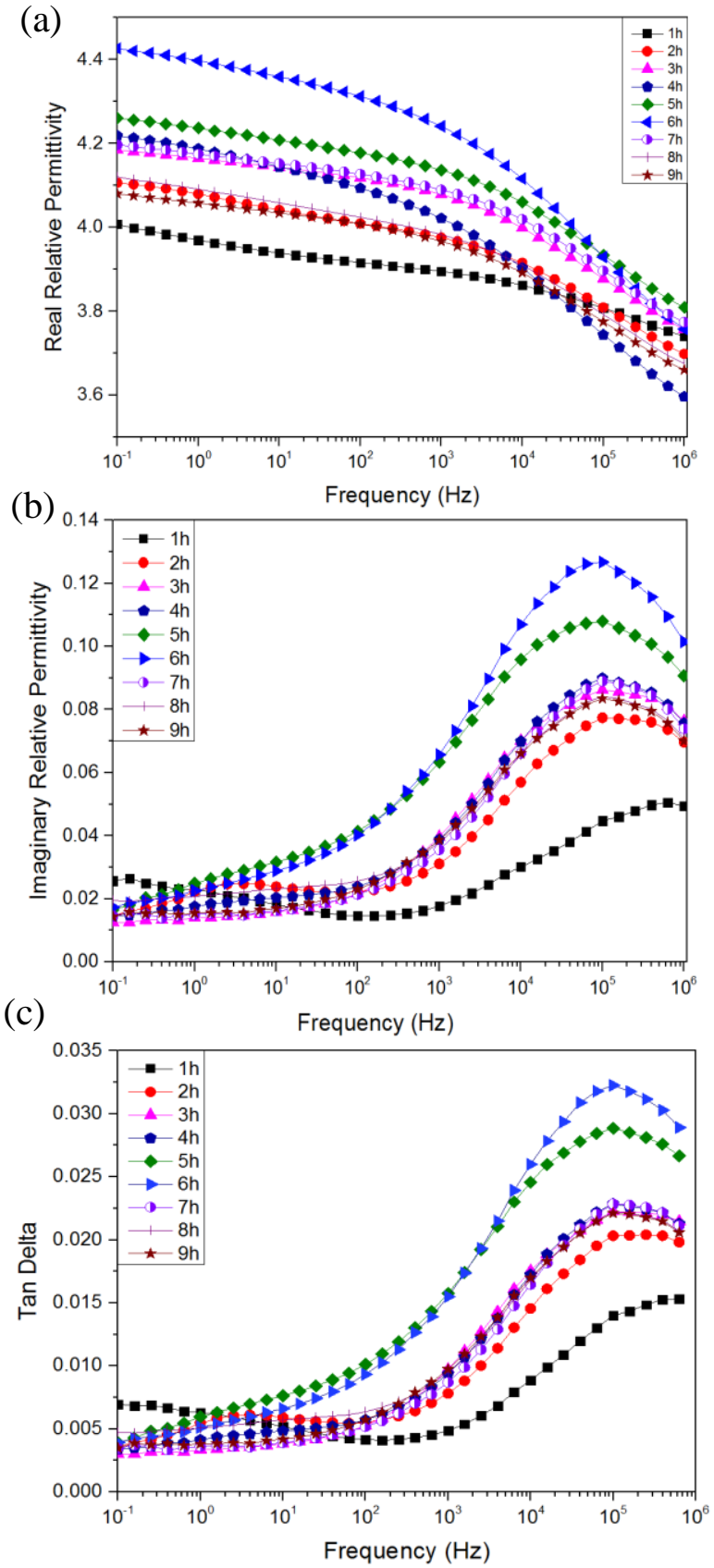

Fig. 2. Dielectric properties of epoxy resin systems cured at different curing hours: (a) real part of complex permittivity, (b) imaginary permittivity of complex permittivity and (c) Tan Delta. 
The dielectric $\alpha$-relaxation at frequency below $100 \mathrm{~Hz}$ of imaginary permittivity also change with increasing the curing time as shown in Fig. 2 (b). This behavior is expected as $\alpha$ relaxation is linked to $\mathrm{T}_{\mathrm{g}}$ [5], and the noted variation in $\mathrm{T}_{\mathrm{g}}$ could have resulted in the variation in $\alpha$-relaxation.

Compression between cured and post cured samples indicated that post cured samples have lower real permittivity and higher losses, as shown in Fig. 3. However, it is evident that post curing time had only limited influence on the dielectric response of the post cured samples.

(a)

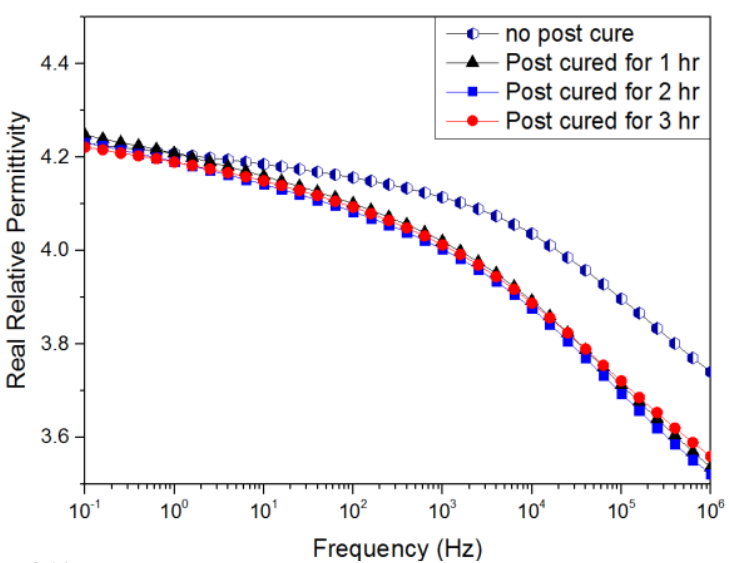

(b)

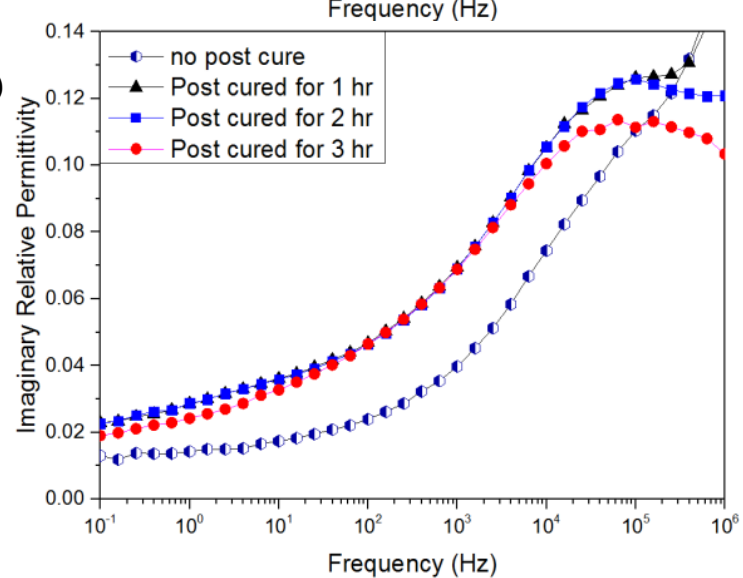

(c)

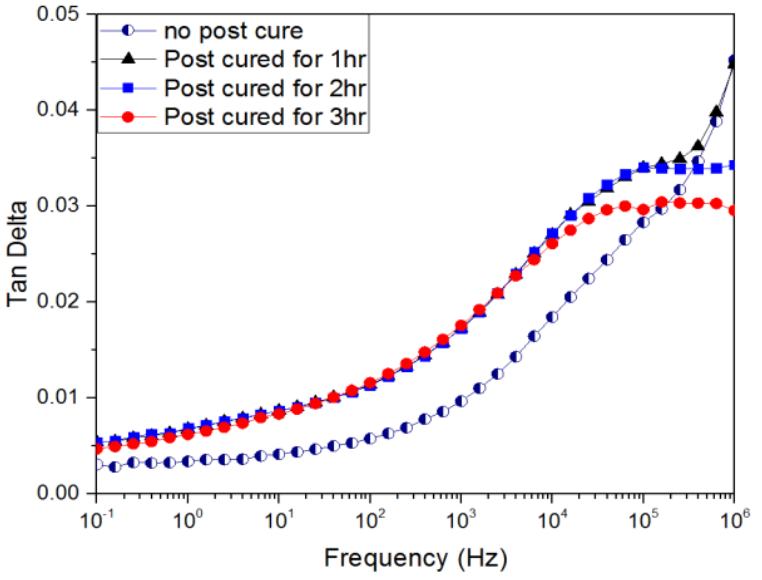

Fig. 3. Dielectric properties of epoxy resin systems. (a) real part of complex permittivity, (b) imaginary permittivity of complex permittivity and (c) Tan Delta of epoxy resin samples post cured at different post curing time.

\section{AC Breakdown test}

Fig. 4 shows Weibull parameter $\alpha(\mathrm{kV} / \mathrm{mm})$ of the $\mathrm{AC}$ breakdown strength of epoxy resin samples cured at $80{ }^{\circ} \mathrm{C}$ for times ranging from $1 \mathrm{~h}$ to $9 \mathrm{~h}$. Unexpectedly, these results indicate that the breakdown strength of samples cured for just $1 \mathrm{~h}$ have the highest measured breakdown strength, around $125 \mathrm{kV} / \mathrm{mm}$. Initially, as the curing time increased, the breakdown strength decreased gradually until, after $3 \mathrm{~h}$ of curing, it increased again. Comparing this behavior with the conductivity indicates that conductivity of the samples tends to have the opposite trend, as indicated in Fig. 5. Although it is not possible to provide a definite explanation for this, some speculation is nevertheless possible. Kumar et al. [6] studied the curing behavior of epoxy resin and showed that the crosslink density is a strong function of the curing time. Therefore, it might be possible that the cross link density could have influenced the variation in the breakdown strength.

As the systems become post cured, the effect of the time of post-curing become limited. Table I along with Fig. 6 shows the probability of $\mathrm{AC}$ breakdown strength of sample cured at $80{ }^{\circ} \mathrm{C}$ for $2 \mathrm{~h}$ then post-cured at $125^{\circ} \mathrm{C}$ for $1-3 \mathrm{~h}$. The results indicate that samples post cured for $2 \mathrm{~h}$ have higher breakdown strength compared with system that was post-cured for $1 \mathrm{~h}$. However, post curing for $3 \mathrm{~h}$ has only limited effect on the breakdown strength, where the $90 \%$ confidence bounds of samples post cured for $3 \mathrm{~h}$ overlap for low probability of failure (at less than 5\%).

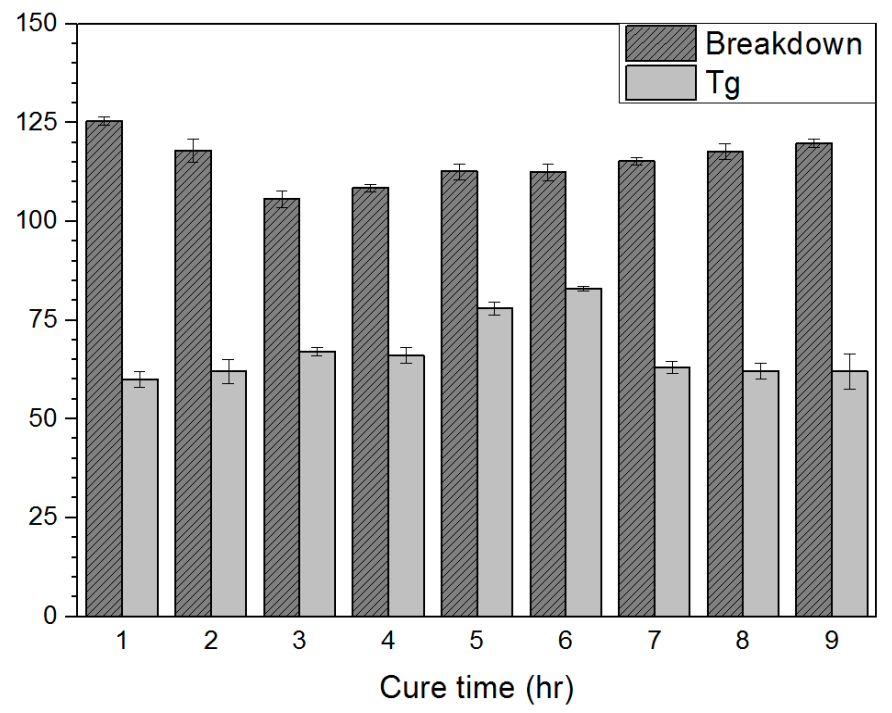

Fig. 4. Breakdown strength and glass transition temrpature data obtained for neat epoxy resin samples cured from $1-9$ hours at $80^{\circ} \mathrm{C}$

TABLE I. AC BREAKDOWN STREGNTH DATA OF POST CURED SAMPLES

\begin{tabular}{|c|c|c|}
\hline Sample ID & Weibull $\boldsymbol{\alpha}(\mathbf{k} \mathbf{V} / \mathbf{m m})$ & Weibull $\boldsymbol{\beta}$ \\
\hline 1 hour of post cure & $107.8 \pm 1.5$ & 23.1 \\
\hline 1 hour of post cure & $121.9 \pm 1.4$ & 26.6 \\
\hline 1 hour of post cure & $129.9 \pm 2$ & 19.7 \\
\hline
\end{tabular}




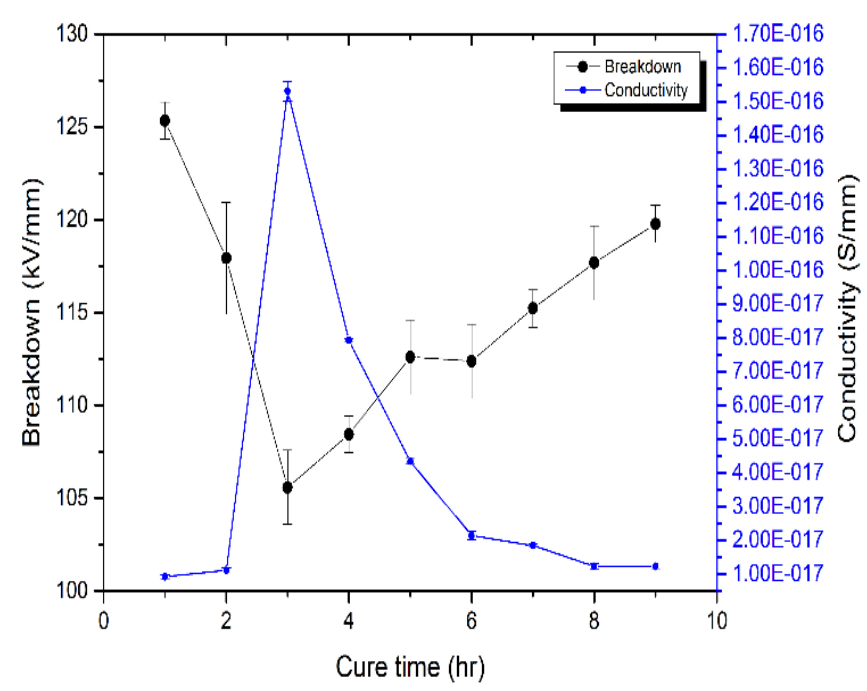

Fig. 5. Weibull parameter $\alpha(\mathrm{kV} / \mathrm{mm})$ of the AC Breakdown strength vs conductivity for neat epoxy resin samples cured from $1-9$ hours at $80^{\circ} \mathrm{C}$

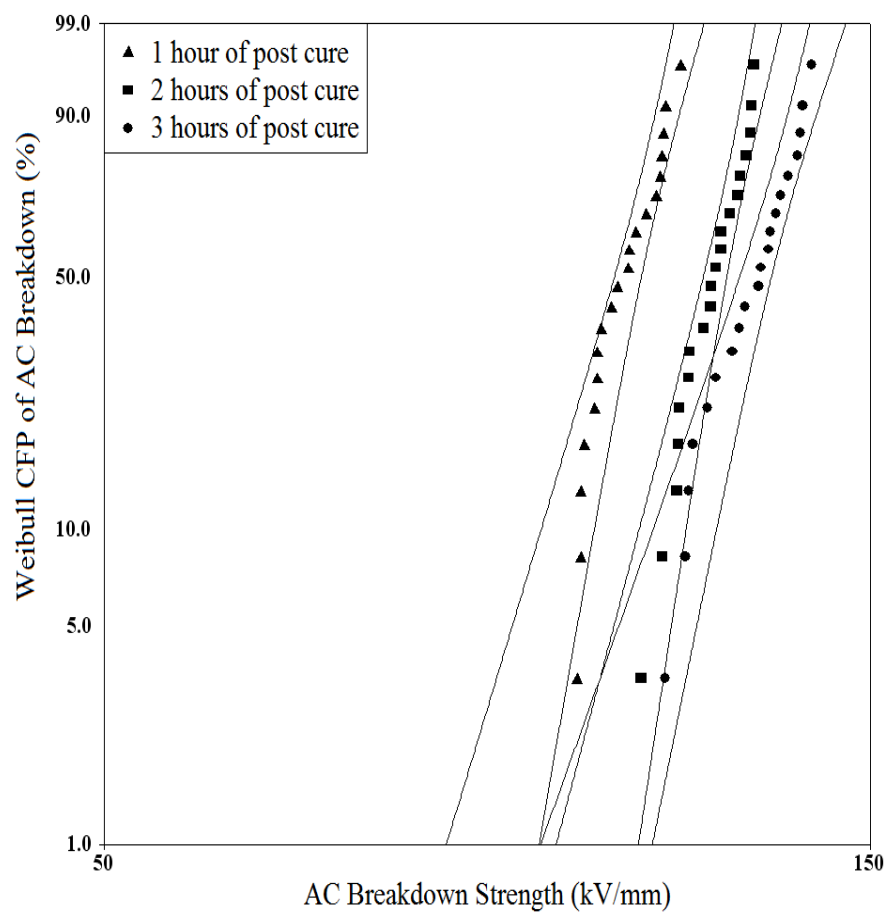

Fig. 6. Weibull plot of breakdown data obtained for neat epoxy resin samples cured from 2 hours at $80^{\circ} \mathrm{C}$ then post-cured at $125^{\circ} \mathrm{C}$ for 3 hours

\section{CONCLUSIONS}

The effect of curing time on the electrical and thermal properties of neat epoxy resin systems has been studied. It was found that both $T_{g}$ and the permittivity of cured samples are highly effected by changes in curing time. The dielectric $\alpha$ relaxation was varied associated with the change in $T_{g}$, whereas the peak of $\beta$-relaxation was suggested to be controlled by the number of crosslinks; however, as reaction proceeds it become environmentally constrained. It was also shown that the breakdown strength initially decreased gradually as the curing time increased but that, after $3 \mathrm{~h}$ of curing, the breakdown strength increased again. However, the DC conductivity of the samples tends to exhibit the opposite trend.

In addition to studying the effect of curing time, the effect of post curing time on the electrical properties was also investigated. It was found that post curing is beneficial for improving the breakdown strength and dielectric response of epoxy resins. However, increasing the post curing time has only limited influence on the electrical properties of the post cured epoxy resin systems.

\section{ACKNOWLEDGMENT}

The authors would like to thank the Schlumberger Foundation Faculty for the Future for the financial support.

\section{REFERENCES}

[1] M. T. Aronhime, X. Peng, J. K. Gillham, and R. D. Small, "Effect of time-temperature path of cure on the water absorption of high $\mathrm{Tg}$ epoxy resins," J. Appl. Polym. Sci., vol. 32, no. 2, pp. 3589-3626, 1986.

[2] N. D. Danieley and E. R. Long, "Effects of curing on the glass transition temperature and moisture absorption of a neat epoxy resin,” J. Polym. Sci. Polym. Chem., vol. 10, no. 19, pp. 2443-2449, 1981.

[3] T. D. Chang, S. H. Carr, and J. O. Brittain, "Studies of epoxy resin systems: Part B: Effect of crosslinking on the physical properties of an epoxy resin," Polym. Eng. Sci., vol. 22, no. 18, pp. 1213-1220, 1982.

[4] M. M. Coleman, P. C. Painter, and J. F. Graf, Specific interaction and the miscibility of polymer blends. Pennsylvania: CRC Press, 1995.

[5] T. Andritsch, "Epoxy Based Nanodielectrics for High Voltage DC Applications: Synthesis, Dielectric Properties and Space Charge Dynamics," Doctoral dissertation, Delft University of Technology, 2010.

[6] D. S. Kumar, M. J. Shukla, K. K. Mahato, D. K. Rathore, R. K. Prusty, and B. C. Ray, "Effect of post-curing on thermal and mechanical behavior of GFRP composites," IOP Conf. Ser. Mater. Sci. Eng., vol. 75, no. 1, 2015. 